\title{
On the motivation behind MCDA and GIS integration
}

\author{
Karim Lidouh \\ Computer and Decision Engineering (CoDE) Department, \\ Université libre de Bruxelles, \\ Brussels - B-1050, Belgium \\ E-mail: klidouh@ulb.ac.be
}

\begin{abstract}
This contribution is a summary of several conclusions drawn from discussions with some of the speakers and participants of the MCDA '74 Meeting (October 2011, Yverdon-les-Bains, Switzerland). In it, we explore the reasons for integrating MCDA and GIS and give an overview of the concrete works that have been achieved, the tools that are available and their use. This is not meant to be a state of the art in this domain. It is merely an analysis of the results that have been accomplished and how these position themselves in the general objective that is the MCDA-GIS integration. From a technical point of view to the usability that is expected from integrated systems, we will see what drives this work and the types of results that one can or should expect.
\end{abstract}

Keywords: multicriteria decision aid; MCDA; geographical information system; GIS; MCDA-GIS integration.

Reference to this paper should be made as follows: Lidouh, K (2013) 'On the motivation behind MCDA and GIS integration', Int. J. Multicriteria Decision Making, Vol. 3, Nos. 2/3, pp.101-113.

Biographical notes: Karim Lidouh has a degree as a Civil Engineer in Computer Science and completed a Master in Management. In 2007, he started research on the integration of multicriteria tools in geographical information systems. He works as a Teaching Assistant at the Solvay Brussels School of Economics and Management (SBS-EM) in the fields of statistics and quantitative methods. He also gave occasional courses on operations research at the IESEG School of Management.

This paper is a revised and expanded version of a paper entitled 'Intégration d'outils multicritères dans les systèmes d'information géographique' presented at 73rd and 74th Meetings of the European Working Group on Multi-criteria Decision Aiding, Yverdon-les-Bains, Switzerland, 6-8 October 2011. 


\section{Introduction}

Why should we integrate? Since the start of the 90s, research works have been studying the conjoint use of spatial analysis methods and multi-criteria methods (Chakhar and Martel, 2003; Chakhar, 2006; Eastman et al., 1993; Greene et al., 2011; Laaribi, 2000; Malczewski, 1999, 2006). The reason behind it being that an increasing number of problems presented characteristics from both fields. Indeed, most spatial decision problems apply to existing situations with several stakeholders and several factors that need to be taken into account. Geographical information systems (GISs) have always been considered as decision support systems because of their data management, analysis, and visualisation functionalities. However, on their own, they lack the analytical functionalities to take several factors into account simultaneously and produce multivariate results. Introducing multi-criteria aggregation functions, sensitivity tools, and methodologies (Bouyssou et al., 2006) in general can therefore help improve the analyses that are made of spatial decision problems.

During the last 20 years, the integration attempts made remarkable progress. Programmes have been developed to allow the conjoint use of the two disciplines, new methods were designed to take care of specific geographic and multi-criteria questions, some methods have been adapted and used on problems that were not initially targeted, and the world of spatial information systems has opened up to multi-criteria analysis as a new way to aggregate sources of data.

We could stop at this, but we would not be entirely persuaded that there really is a need to integrate these two fields. Certainly, an integrated system is always useful, but there are many ways to solve these types of problems without resorting to an integrated system. Up till now the systems that have been developed can be placed into several technical levels of integration ranging from weak coupling to fully integrated systems (Chakhar and Martel, 2003; Laaribi, 2000). Surprisingly however, the solutions with the greater success are the ones that rely on weak coupling (Malczewski, 2006).

This makes us wonder about the real usefulness of this integration and there is now a real discussion as to whether it is necessary to continue the work even further than the solutions that already currently exist. For now, we do not know exactly what this integration is exactly needed for and if it really is interesting to invest several months of work in the development of such a solution. To find an answer to this problematic, it is necessary to focus on what has been accomplished already, on the types of problems that have been studied, and on what could be improved within the process of these analyses and the way they are conducted.

Section 2 of this paper describes the integration of MCDA and GIS, the types of solutions that can be developed, as well as their advantages and inconveniences. The aim is not give an entire state of the art of the subject but well to raise the different factors that will affect the conjoint usage of MCDA and GIS. The interested reader can refer to Malczewski's (2006) exhaustive review or several other works (Chakhar, 2006; Greene et al., 2011; Laaribi, 2000) to get an idea of the current situation and conjoint uses of MCDA and GIS. In Section 3 we explore the technical aspects and difficulties of the integration and illustrate it with examples of existing tools and software. We also see some development options that are available for people wanting to programme a custom integrated solution. Finally, Section 4 is a study proposal on the usability of integrated systems, the target audience, the needs that should be met. 
This future study should be the most important contribution one could bring to this domain. It would help better orient the efforts that are being made in order to quickly attain better results.

\section{The integration of MCDA and GIS}

In this section, we will cover the characteristics and advantages of the different levels of MCDA-GIS integration. Our aim is to understand when each of these solutions is used and with what goal. Of course the following classification is not crisp as there can be solutions that share similarities with different levels of integration.

\subsection{Weak coupling}

Loose coupling is the first usage that has been made of GIS and MCDA-capable systems. It consists in using specific programmes or tools for the different steps of the analysis. The communication between these programmes, which can be complex at times, is handled by the analyst. A common decision process could resemble the following steps:

- defining the alternatives using the GISs data management functionalities (e.g., binary or overlay operations)

- evaluating the alternatives by entering their characteristics in an MCDA application

- $\quad$ taking knowledge of the results and producing a map to display them using a GIS.

The direct advantage of this procedure was its low development cost. However this came at a heavy price as the concrete separation between these steps made it hard to fully comprehend the spatial aspect of the problem. Spatial relationships between alternatives were therefore not considered at all during the MCDA evaluation step. Furthermore since the transfers of data were done by human interventions, the risk of errors was high. And in the cases where interfaces were developed to link different systems and transfer data automatically from one another, the works achieved could not be used with other systems because of technical differences.

\subsection{Tight coupling}

In the last ten to 15 years, the need for automatic communications between systems has given birth to works that coupled MCDA tools and GIS under a single interface. To achieve such a result the methods of one field (usually MCDA) were implemented as a module, routine or script into the other system which is used as a base. Even though the sources of data were not always the same and the data transfers from one system to another were not entirely transparent to the user, this type of tight coupling has been an important step towards the integration of both fields. By making both tools known to the analysts and easier to use, these systems showed the importance of combining them. A few drawbacks were still present though. Flexibility and interactivity were the main concerns of these systems. Researchers began therefore to search for ways to 
break multi-criteria decision processes in order to give analysts the same freedom they experience with spatial analysis functions (Chakhar and Martel, 2003).

\subsection{Full integration}

When looking at the evolution of information technologies, we see that through the internet, several applications and services have opened themselves to the masses. Sometimes simplified, they were made available as web services to all individuals and thereby formed a new generation of participatory systems. These are characterised by their accessibility but also by the way they interact. Web-services nowadays are standardised so that mash-ups (i.e., hybrid combinations of web applications) have been made possible. Their evolution into linked services is reaching a new stage in the type of experience they offer to the user (Pedrinaci and Domingue, 2010). The number of websites that use Google Maps as a base and add services on top (e.g., weather, navigation, traffic, parking...) has recently experienced very strong growth. Of course one could expect the same type of user experience from multi-criteria spatial decision support systems. This however would require a good formalisation of all data structures used in these kinds of problems. Standards have started being defined for geographical information, yet there is, apart from the XMCDA encoding standard elaborated by Decision Deck (http://www.decision-deck.org) Consortium, hardly anything similar available for multi-criteria techniques.

Ideally a fully integrated MCDA-GIS system should offer multi-criteria functionalities and spatial analysis functionalities and allow the user to access any of those at any given time during an analysis. The user should also be able to interact with the different processes by changing the parameters of the methods and visualise the results or the different spatial elements of the problem directly on a map.

\subsection{Technical aspect vs. operational aspect}

The separation that we have just seen in three levels of integration has been made while keeping the technical aspect in mind as that has been the main concern for these past years. We should however be careful not to forget the operational aspect of such systems. Indeed, the distinction between levels of integration can be made based on the technology used for the system, but it could also be used to measure the usability offered by it.

When we consider these two aspects, we can easily understand why the higher levels of integration suffer from a lack of interest while the lower levels thrive. Indeed, even nowadays, for most novel studies that mix MCDA and spatial analysis, the researchers prefer to resort to separate programmes instead of using integrated solutions. It could be due to a lack of available systems or knowledge thereof (most integrated solutions are rarely known to the analysts that need them). However the most plausible explanation could be related to the usability of these systems and the place they can take in an analysis. When considering an integrated system, unless the solution perfectly matches the problem at hand, the user will always try to couple it with separate tools that better fit the tasks to be done.

When an analyst takes on a new problem, its characteristics are rarely explicitly defined. The analyst must therefore proceed by exploring the properties of the decision 
problem before modelling it. Oftentimes, no system will be able to replace the analyst's expertise on the matter. Furthermore a fully integrated system that has been designed to follow general decision support procedures will not always have the flexibility to allow small changes in the process to adapt to a very specific problem. We could therefore summarise the situation as follows ('+' signs indicate advantages, '-' signs indicate inconveniences):

Weak coupling:

Uses separate tools or programmes for the different parts of the analysis that are able to export-import data from one another.

- Technically the weakest integration.

- Error-prone.

- Not iteration friendly.

+ Offers the most freedom to the user (i.e., analyst) and relies on his/her experience.

\section{Full integration:}

Uses a single system that proposes several preselected functionalities to deal with most commonly encountered problems.

+ Better communication of data.

+ Makes application of processes easier.

+ Allows interactions during the analysis.

- Forces the user to work with predetermined methods or tools.

Now that we have identified the strong and weak points of the two different approaches, we can now take interest in the existing solutions for such implementations. Furthermore, by taking interest in the way that integrated systems are used we should be able to identify the shortcomings on which to work to fulfil the needs of the users.

\section{Technical aspects of the integration}

In the following section, we will illustrate these technical levels of integration while trying to identify the associated operational level of integration. These examples will show us that unfortunately, even though impressive technical advances have been made, the operational aspect has up till now been neglected.

\subsection{Existing MCDA-GIS software}

Since the start of the integration works, several options of MCDA-GIS systems have been proposed. But out of those, very few have survived the test of time. Indeed, most developments by researchers have either been forgotten and never updated or were never made public. That is the case for most solutions that were developed in the framework of private projects. In the end, the solutions that stayed in use and are still available today are the one that were backed up by powerful commercial platforms or active communities.

Initially developed for research and scientific purposes, IDRISI GIS has rapidly grown to become an important actor in the field of GISs. As such, an impressive number of modules have been added to it since its debut and it was the first commercial GIS to ever integrate routines for the SMART methodology and for the determination of the weights using Saaty's method. These routines were well documented (Eastman et al., 
1993) and the authors also proposed examples of usage on raster as well as vector data. Later extended, the current version of the software includes a complete MCDA module with support for the ordered weighted average (OWA), MOLA heuristic, and analytical hierarchy process (AHP).

Figure 1 IDRISI GIS' interface (see online version for colours)

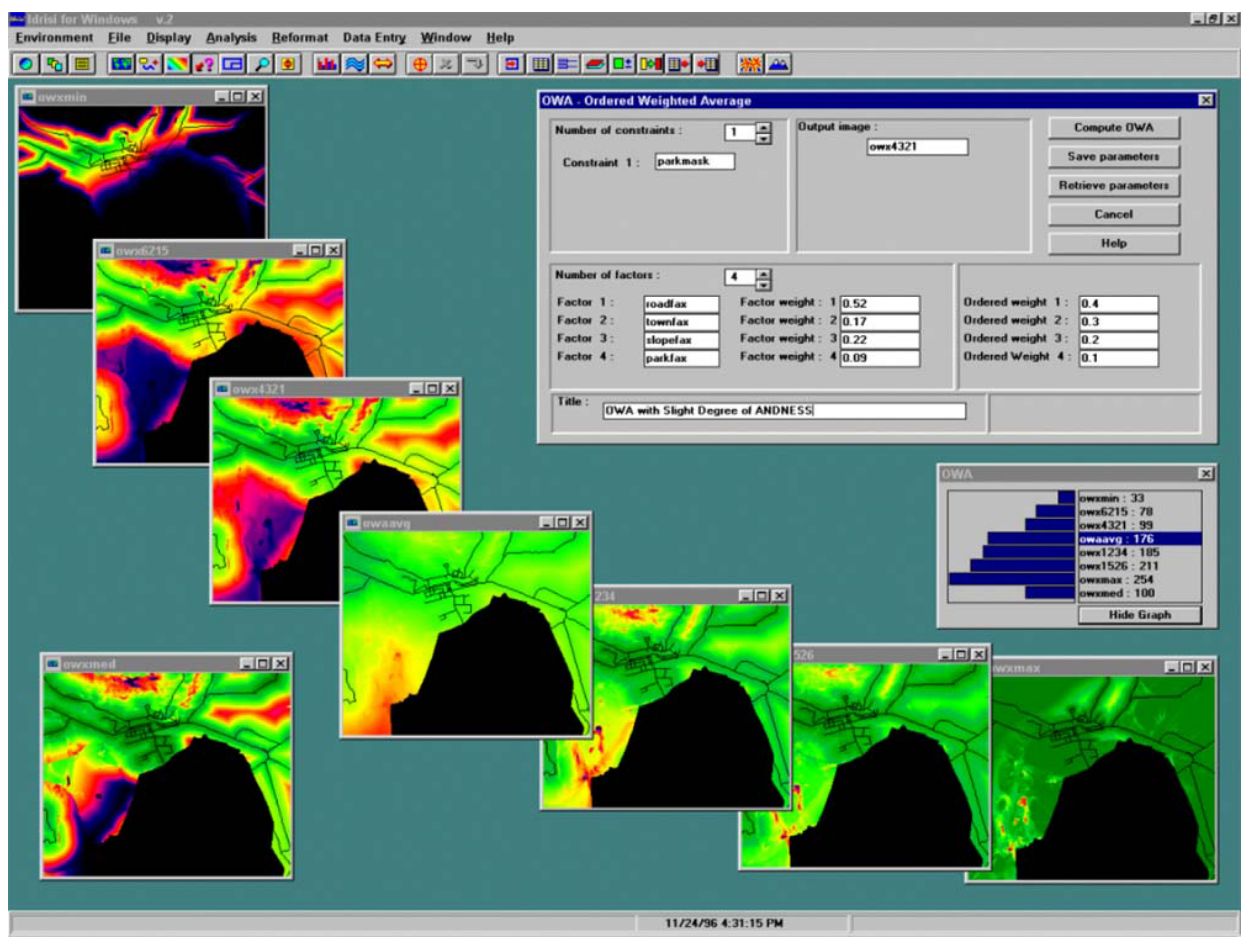

Source: IDRISI GIS (http://www.clarklabs.org)

Of course, we could not talk about commercial GIS without mentioning the most widely used software suite. ESRI's ArcGIS software suite is now one of the best known alternatives on the market. Proposing systems for virtually every type of application requiring the management of geographic information, ESRI has put a great effort into making its systems very accessible. The ArcScripts section of their website has now for a long time allowed developers to propose their own functions and additional packages to complete the existing systems. Among those scripts, one can find that several methods are available: AHP, OWA, SAW, TOPSIS ... ArcScript is unfortunately not open anymore for new submissions, but ESRI has added several other services in replacement. This behaviour is typical of most software platforms, which offer the possibility of extending their functions to attract user communities.

A third system that deserves praise is the DECERNS SDSS, a project being developed at the IATE (Obninsk University, Russia) and integrating MCDA and GIS in a single web-based architecture (Sullivan et al., 2009). Since they share the same database, this system allows the user to rapidly switch to any tool at any moment of the analysis. They can thereby engage in a more iterative process of testing the robustness 
of a model and changing the parameters before giving final recommendations. The system implements an impressive number of MCDA methods (e.g., MAVT, MAUT, AHP, TOPSIS, PROMETHEE, SMAA, FlowSort ...) and support tools (e.g., walking weights, line weights, sensitivity analysis ...). However, even though both tools have been developed together, they were kept separate as two independent subsystems that are able to interact. This system is therefore technically a complete integration, but behaves like a tight coupling. Indeed, from the user's view point, the two tools seem separate as he/she has to change interfaces to use either one. Yet, some improvements are planned which means that DECERNS might very well become one of the first complete systems to provide a full integration experience for the users. Figures 2 and 3 show screenshots of the decision-making support (DMS) subsystem and the GIS subsystem.

The last three systems were examples of integrated systems, but there are other options to solve a decision problem using MCDA and a GIS. As we said earlier, most analysts resort to loose coupling solutions to do preliminary analyses of the problems if not entire studies. For problems that do not require too complex analytical functions, there is indeed the possibility of coupling separate software for the different steps of the analysis. Analytical software such as R, Mathworks MATLAB, and Microsoft Excel have since long been included in spatial analyses with some of the major GIS. For that purpose, most of them are able to export their attribute data in some of the most popular formats such as Excel or CSV.

Figure 2 DECERNS SDSS' DMS subsystem (see online version for colours)

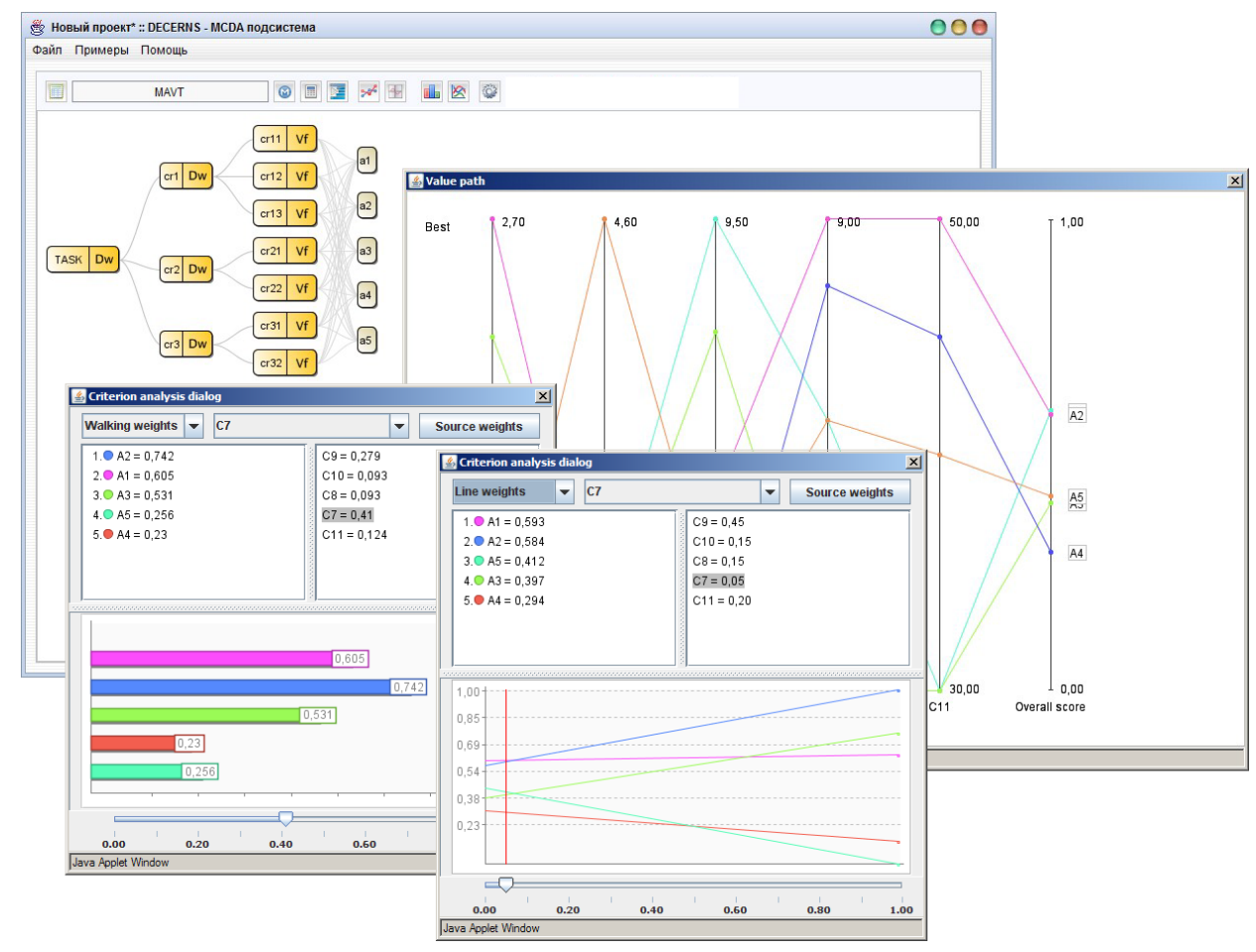

Source: DECERNS SDSS (http://www.decerns.com) 
Figure 3 DECERNS SDSS' GIS subsystem (see online version for colours)

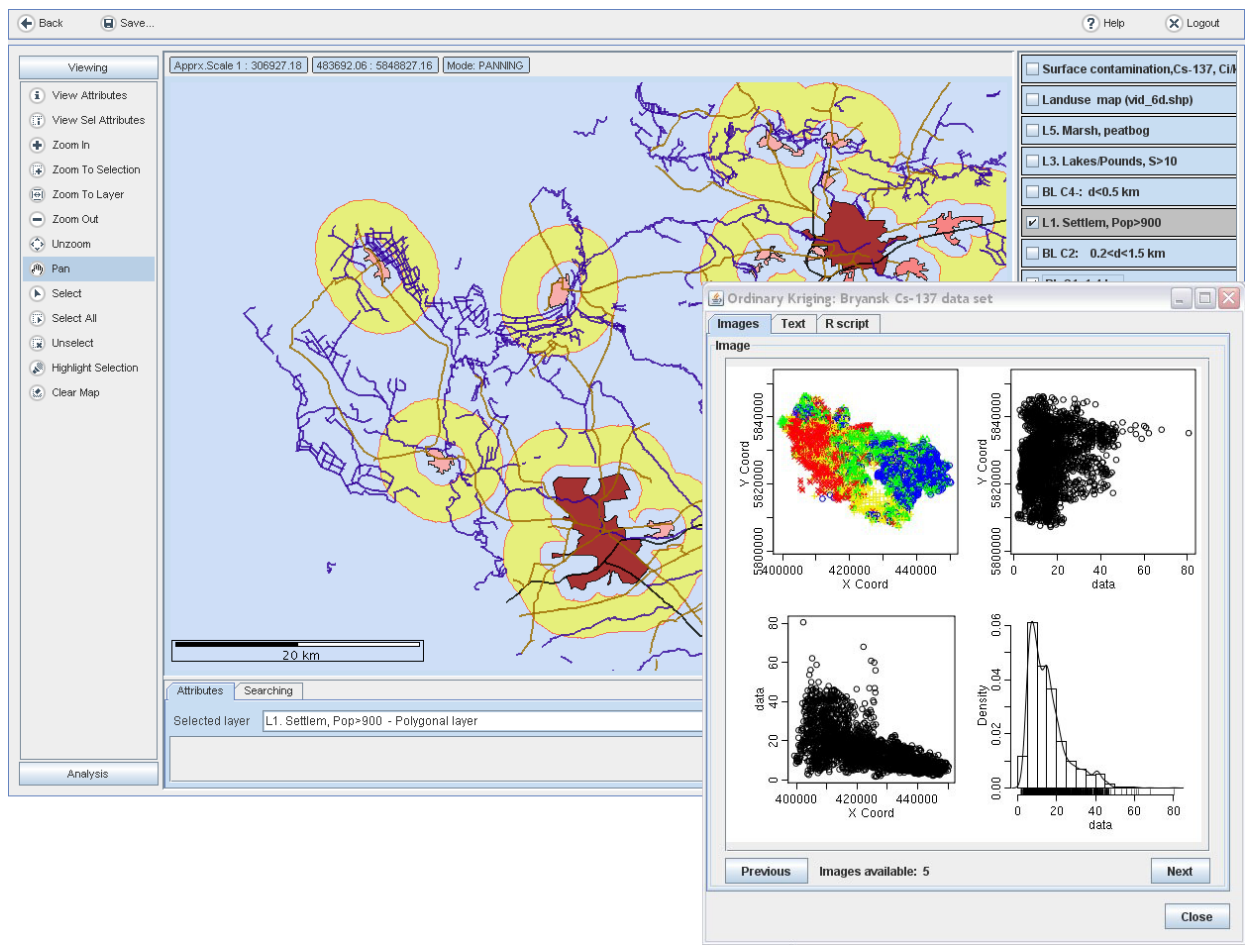

Source: DECERNS SDSS (http://www.decerns.com)

\subsection{Existing spatial or MCDA tools}

Aside from the already integrated MCDA-GIS solutions, there are several tools, libraries, and software parts that could be used to produce a working MCDA-GIS solution for a specific purpose. As we have just seen earlier, most GIS are able to extend their functionalities using scripts, but that is not the only way.

Systems like GRASS GIS, an open source desktop software, offer the possibility to the users to develop modules or plug-ins to enhance the already impressive offer of functionalities. After getting used to its complex interface, one can discover the most complete open source GIS with support for a large panel of formats. Other examples of extensible GIs are ESRI's ArcGIS, QuantumGIS, SAGA GIS.

Some commercial GIS such as ESRI's ArcGIS or Microsoft's MapPoint are also available as APIs (i.e., application programming interfaces) which are components to be used to add GIS functionalities to almost any programme one could have done on its own. It is thereby possible to add map visualisation possibilities or management of spatial data or even access to some spatial analysis functions. Finally these APIs can also be independant from any complete GIS software. GeoTools and GeotoolKit are examples of libraries that can be used to add geographic functionalities to existing development projects.

On the other hand, when we consider MCDA components for development. There are almost none. The only available libraries are now part of Decision Deck 
(http://www.decision-deck.org), a group of initiatives acting to offer an open source platform for developing, designing, and sharing multi-criteria tools.

\subsection{Technical difficulties encountered}

Even though so many tools exist to help us with the integration, there are still some pieces missing. Indeed, there are little to no MCDA open source libraries or software parts. Also the semantic differences between the two fields or within each field make it difficult to conceive a unique system with both types of functionalities. It is indeed necessary to agree on a common language to be used in this type of integration.

Also, since the MCDA functionalities are more likely to be integrated in a GIS than the opposite (Malczewski, 2006), there is a need to select some specific methods from the multi-criteria field and find ways to help a user decide on which one to use and how to do that. Several works have focused on the choice of an MCDA method depending on several characteristics of a problem (decision problematic, type of alternatives, ...) but unfortunately there have not been any implementations that were made public.

\section{Usability aspects of the integration}

As already stressed by Malczewski (2006) in all the works that have been realised on MCDA-GIS integration, there is a lack of usability studies aimed at expressing the needs these systems should fulfil. In this section, we aim to clear the ground and lay the foundations for such usability studies. Among others, we will ask ourselves the following questions: who will be the users of such systems and what will be their goals which such tools?

\subsection{The users}

In order to better assess the needs that integrated systems have to fulfil, it is necessary to ask ourselves who will be their users. And the question is not that easy to answer either because there can be several stakeholders with different profiles in a decision problem. It seems obvious that the ones that will most benefit from an integrated MCDA-GIS system are of course the analysts that are familiar with MCDA and spatial analysis. But let us not forget all the other roles in a decision problem. We have the experts that can offer their help in evaluating alternatives or parameters for the methods. We also have the decision makers that have to indicate their preferences and give an idea of where their interest lies.

These are thus the main users we can encounter in a typical decision problem:

- the analyst: has extensive knowledge of the MCDA and spatial methods used and is in charge of modelling the problem as well as giving the final recommendations to the decision makers

- the experts: are in charge of providing the data for the effective comparison of the problems alternatives 
- the decision makers: give information on their way of thinking and the aim they pursue and expect an understandable report on the decision they need to make and the reasons behind the obtained results.

Naturally a complete study would not stop at this, but would involve characterising user groups for each of the previous roles by analysing:

- who the end-users are (profiles, knowledge of the methods used...)

- which tasks they perform with GIS and MCDA in order to achieve specified goals, when and where they use the different functionalities of such a system

- the way they react and what they expect from the usage of a MCDA-GIS platform.

In order to collect data, one could then make use of the usability checklist developed by Johnson et al. (1989) or some of the later works by Davies and Medyckyj-Scott (1996). It would allow us to evaluate MCDA-GIS user interface mockups in terms of effectiveness, efficiency, and user satisfaction. On a more amusing note, one can notice that Johnson's studies tend to evaluate the users' perception of the system based on several criteria. The aim however is absolutely not to choose, rank or sort anything (i.e., the typical objectives of MCDA aggregation methods), but merely to evaluate the different aspects of a given user interface as well as the system behind it.

\subsection{The MCDA-GIS problem solving process}

Now that we know which roles we have to pay attention to, taking a deeper look into the entire decision process for a problem may help us identify the needs of all these persons as well as the kinds of systems that will be able to cover them.

Several researchers have proposed concepts of flowcharts for decision processes, starting with Malczewski (1999) in an attempt to identify the tools that were most needed for each step of the analysis. Figure 4 shows a flowchart in three phases that are composed of the following steps:

1 Intelligence phase: the analyst gathers data on the problem in order to determine the type of method to use and stores the information in the GIS.

- Problem definition: this step defines the objective of the process. It consists in choosing a particular decision problematic (choice, ranking, sorting, description).

- Evaluation criteria: since some of the criteria might be spatial, the analyst can use the GIS to define them with the help of experts.

- Constraints: since the alternatives for these problems will be spatially-referenced, the analyst can use the GIS to define a number of constraints that delimit them.

2 Design phase: after gathering all the needed data, the analyst establishes the model for the decision problem, in a second phase that will mostly rely on multi-criteria functionalities. 
- Alternatives: once the constraints have been entered if any, the analyst can generate a list of alternatives or define the set of feasible solutions.

- Decision matrix: when both the criteria and the alternatives have been defined, the analyst can start filling the evaluation table with the help of experts if necessary. In some cases, if all the necessary data had been gathered before, this step could be automatic.

- Decision maker's preferences: at this point the decision maker can indicate his view of the problem and help the analyst enter the parameters for the chosen method.

- Decision rules: depending on the method used, there might be a need to resort to decision rules too.

3 Solution phase: finally, once the model has been completed, can it be used to explore the set of feasible solutions, usually in company of the decision maker.

- Sensitivity analysis: this step will serve to verify the robustness of the obtained solution or to adjust the model according to the decision maker's wishes.

- Recommendation: at the end of the analysis, the analyst is expected to provide an answer and its justification to the decision maker concerning the problem and explain how to implement it.

Figure 4 Decision flowchart
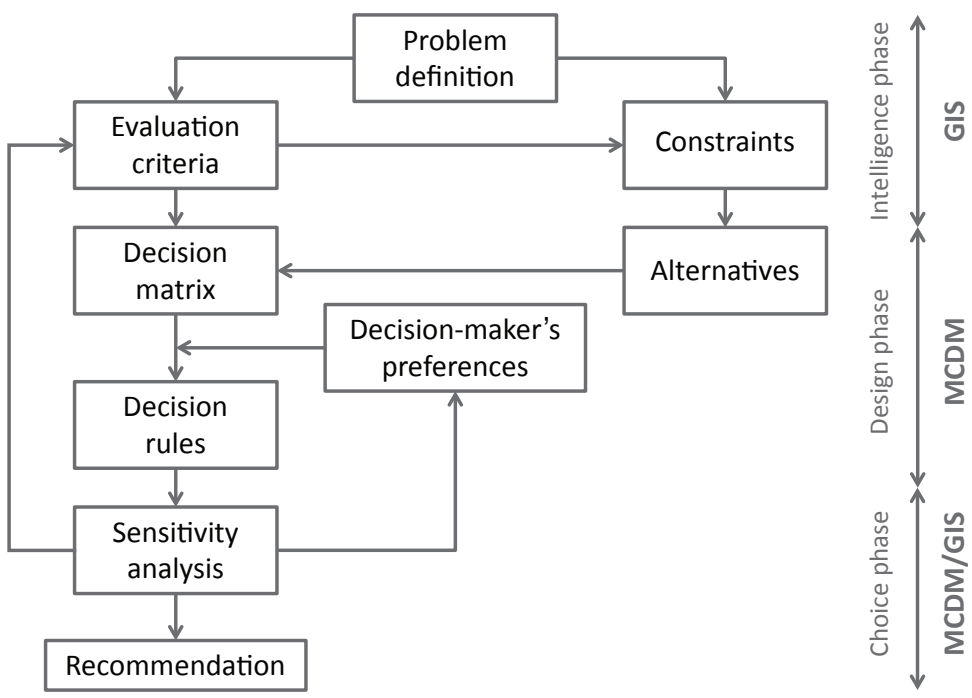

Source: Adapted from Malczewski (1999)

There are of course other flowcharts that have been proposed more recently by other researchers (Chakhar and Martel, 2003), but this one already tells us a lot on what needs to be done by each person involved. 


\subsection{The importance of an integrated system}

From the previously analysed flowchart, we can tell that up till now only the first two phases have been correctly implemented in the existing systems. Indeed those two phases only serve to establish the decision model and obtain an initial solution. That can be done easily in tightly coupled solutions such as a MCDA module in a GIS.

To further explore the possible solutions with the decision maker would require an integrated interface that allows for changes in the parameters while keeping the map displayed and constantly updated. Interactivity is what could be the most valuable function in an integrated system. some works on visualisation could help with this aspect (Andrienko and Andrienko, 2006; Bertin, 2010; Lidouh, 2011).

Unfortunately with the few existing systems that currently allow it, one must settle for separate interfaces that create a transition between one scenario and the next with some modifications. It is currently not possible for MCDA modules to interactively change the displayed results on a map by modifying the parameters of a method.

Finally, let us not forget that the usual MCDA problematics are not the only ones that matter in this field. There are also several analysis techniques that couple MCDA and spatial aspects to produce results that support a decision. For example some methods allow you to segment a territory based on several criteria (Tavares-Pereira et al., 2007), to study the evolution of decision maps (Takougang, 2010), or even to compare different maps according to several criteria. All of these can be used to help the analyst at several steps of the analysis. Unfortunately though, there is not yet to our knowledge a complete list of these types of works since they are quite recent.

\section{Conclusions}

In this contribution, we took an interest in two fundamental aspects of the MCDA-GIS integration: the technical aspect and the operational aspect.

We have explored the current offer in the MCDA-GIS field. There have been numerous works but very few have lasted until now and continue to be used by geographers or spatial analysts. There is however a lack of multi-criteria tools made for the purpose of helping with developments. Indeed almost no projects are available on the key distribution channels for open source developments.

Integrating the two fields obviously brings together their advantages but also their drawbacks. Where two separate systems offer more flexibility, an integrated system can offer robustness and the possibility for higher interactivity with the decision processes. It is therefore quite difficult to predict which should be used when ignoring the characteristics of the problem to be solved.

A usability analysis should be done to assess the expectations of all the actors in a decision problem and to help them communicate and work together. The brief look we have directed to this aspect has shown us that the analysis and communication of the results can truly benefit from the integration in a single system.

In the end, it seems the question we should ask ourselves is not whether we need to integrate, but how we should do it. In this world where data is becoming much easier to obtain, bringing together different disciplines and finding new synergies are what we should aim for. Thus we need to focus on the usability of the systems and tools that we develop and determine who will be the users and what are their needs. 


\section{References}

Andrienko, N. and Andrienko, G. (2006) Exploratory Analysis of Spatial and Temporal Data, A Systematic Approach, Springer, Berlin.

Bertin, J. (2010) Semiology of Graphics: Diagrams, Networks, Maps, ESRI Press, Redlands, California.

Bouyssou, D., Marchant, Th., Pirlot, M., Tsoukiàs, A. and Vincke, Ph. (2006) 'Evaluation and decision models with multiple criteria: stepping stones for the analyst', International Series in Operations Research \& Management Science, Vol. 86, Springer-Verlag.

Chakhar, S. and Martel, J.M. (2003) 'Enhancing geographical information systems capabilities with multicriteria evaluation functions', Journal of Geographic Information and Decision Analysis, Vol. 7, No. 2, pp.47-71.

Chakhar, S. (2006) 'Cartographie décisionnelle multicritère : Formalisation et implémentation informatique', $\mathrm{PhD}$ thesis, LAMSADE Université de Paris Dauphine, France.

Davies, C. and Medyckyj-Scott, D. (1996) 'GIS users observed', International Journal of Geographical Information Systems, Vol. 10, No. 4, pp.363-384.

DECERNS SDSS, available at http://www.decerns.com.

Decision Deck, available at http://www.decision-deck.org.

Eastman, J.R., Kyem, P.A.K., Toledano, J. and Jin, W. (1993) GIS and Decision Making, UNITAR, Geneva.

ESRI ArcScripts, available at http://arcscripts.esri.com.

Greene, R., Devillers, R., Luther, J.E. and Eddy, B.G. (2011) 'GIS-based multiple-criteria decision analysis', Geography Compass, Vol. 5, No. 6, pp.412-432.

Guitouni, A. and Martel, J-M. (1998) 'Tentative guidelines to help choosing an appropriate MCDA method', European Journal of Operational Research, Vol. 109, No. 2, pp.501-521.

IDRISI GIS, available at http://www.clarklabs.org.

Johnson, G.I., Clegg, C.W. and Ravden, S.J. (1989) 'Towards a practical method of user interface evaluation', Applied Ergonomics, Vol. 20, No. 4, pp.255-260.

Laaribi, A. (2000) SIG et analyse multicritère, Hermès Science Publications, Paris.

Lidouh, K. (2011) 'An adaptation of the GAIA visualization method for cartography', in Computational Intelligence in Multicriteria Decision-Making (MDCM), 2011 IEEE Symposium on, pp.29-35.

Malczewski, J. (1999) GIS and Multicriteria Decision Analysis, Vol. 31, J. Wiley \& Sons, Engineering, New York.

Malczewski, J. (2006) 'GIS-based multicriteria decision analysis: a survey of the literature', Int. J. of Environmental Technology and Management, Vol. 20, No. 7, pp.703-726.

Pedrinaci, C. and Domingue, J. (2010) 'Toward the next wave of services: linked services for the web of data', Journal of Universal Computer Science, Vol. 16, No. 13, pp.1694-1719.

Sullivan, T., Yatsalo, B., Grebenkov, A. and Linkov, I. (2009) 'Decision evaluation for complex risk network systems (DECERNS) software tool', Chapter 12 in: Decision Support Systems for Risk-Based Management of Contaminated Sites, Springer, USA.

Takougang, S.A.M. (2010) 'Contributions à l'aide à la décision en matière de gestion spatialisée. Etude de cas en management environnemental et développement de nouveaux outils', $\mathrm{PhD}$ thesis, Université de Mons - Faculté Polytechnique, Belgium.

Tavares-Pereira, F., Figueira, J.R., Mousseau, V. and Roy, B. (2007) 'Multiple criteria districting problems: the public transportation network pricing system of the Paris region', Annals of Operational Research, Vol. 154, No. 1, pp.69-92. 